\title{
GRAINE project: precise gamma-ray observations with balloon-borne emul- sion telescope
}

\author{
Hiroki Rokujo $^{1, *}$ for the GRAINE collaboration ${ }^{1,2,3,4,5}$ \\ ${ }^{1}$ Nagoya University \\ ${ }^{2}$ Aichi University of Education \\ ${ }^{3}$ ISAS/JAXA \\ ${ }^{4}$ Okayama University of Science \\ ${ }^{5}$ Kobe University
}

\begin{abstract}
Observation of cosmic gamma rays is important in the understanding of high-energy objects or phenomena in the universe. Since 2008, the Large Area Telescope onboard the Fermi Gamma-ray Space Telescope (Fermi-LAT) has surveyed the sub-GeV/GeV gamma-ray sky and achieved high statistics measurements. However, observation at low galactic latitudes remains difficult owing to the lack of angular resolution, and new issues following the operation of Fermi-LAT have arisen. We devised a precise gamma-ray observation project, Gamma-Ray Astro-Imager with Nuclear Emulsion (GRAINE), using balloon-borne emulsion gammaray telescopes to realize high angular resolution, polarization-sensitive, and large-aperture observations in the 10 $\mathrm{MeV}-100 \mathrm{GeV}$ energy region. Following basic developments on the ground, we performed three balloon-borne experiments with upgraded instruments. In this paper, we present results from the second balloon experiment in 2015, a report on the latest balloon experiment conducted on April 26, 2018, and a recent study on hadronic interactions using proton beams.
\end{abstract}

\section{Introduction}

Gamma rays are one of the most important probes to explore the high-energy phenomena in the universe. The information obtained by the observation of cosmic gammarays is useful in various fields, such as cosmic-ray physics, astronomy, cosmology, and fundamental physics. The Large Area Telescope onboard the Fermi Gamma-ray Space Telescope (Fermi-LAT) [1], which is the latest sub$\mathrm{GeV} / \mathrm{GeV}$ gamma-ray detector, has surveyed the entire gamma-ray sky and provided cosmic gamma-ray data with high statistics since its launch in 2008. Until now, several important results were reported, such as the detection of more than 3000 gamma-ray sources [2] or the discovery of the acceleration of cosmic ray protons [3]. However, the observations by Fermi-LAT are severely limited by the lack of the angular resolution. In particular, higher-resolution imaging observation is needed for the detailed understanding of the galactic center region [4], where complex diffuse gamma-ray emissions and many gamma-ray sources exist and extended sources such as supernova remnants are also present [5]. Recently, future projects are proposed, focusing on the improvement of the angular resolution [6-8].

The Gamma-Ray Astro-Imager with Nuclear Emulsion (GRAINE) is a precise observation project of cosmic gamma-rays in the $0.1-100 \mathrm{GeV}$ energy region, using a balloon-borne large-aperture telescope based on nuclear

\footnotetext{
*e-mail: rokujo@flab.phys.nagoya-u.ac.jp
}

emulsion technology [9]. Nuclear emulsion is a tracking detector that can observe three-dimensional trajectories of charged particles with sub-micron resolution and thin material ( $\sim 0.1 \%$ of the radiation length unit). It can precisely determine the incident angle of gamma rays by measuring the starting point of the electron and positron tracks via pair creation $\left(\gamma+(\gamma) \rightarrow \mathrm{e}^{+}+\mathrm{e}^{-}\right)$. The angular resolution of the emulsion gamma-ray telescope is approximately one order of magnitude better than that of Fermi-LAT. Moreover, nuclear emulsions can realize an accurate measurement of the azimuthal angle of the plane where the electron and positron tracks lie, and is sensitive for polarized gamma-rays [10]. Furthermore, the nuclear emulsion is dead-space-free and lightweight. It is the only type of detector that can realize the expansion of aperture area while maintaining the resolution. GRAINE repeats the observation by a balloon-borne experiment with an upgraded detector scale and is expected to be developed into a scientific observation using a full-scale telescope (aperture area of $10 \mathrm{~m}^{2}$ ).

Until now, we have developed various detector components using accelerator beam tests (gamma rays at SPring8 , muons at Muon Pit of the neutrino beam line in JPARC, electrons at linear accelerator, and protons at the Super Proton Synchrotron (SPS) at CERN) [10-14], observations at ground and mountain levels (Norikura) [11, 15], and three balloon-borne experiments. We verified the feasibility of the observation by a small-scale emulsion telescope (aperture area of $0.013 \mathrm{~m}^{2}$ ) in the first bal- 
loon experiment, GRAINE 2011 [16, 17], and then we demonstrated the flight performance using a middle-scale telescope $\left(0.38 \mathrm{~m}^{2}\right)$ in the second balloon experiment, GRAINE 2015 [18-20]. The latest experiment with the middle-scale telescope, GRAINE 2018, was performed in Australia on April 26, 2018, and the flight data analysis is processing.

In this paper, we present the current status of the GRAINE project. Several results of the GRAINE 2015 experiment are shown in section 2. A short report on the balloon flight of GRAINE 2018 is given in section 3. Finally, study of hadronic interactions using the GRAINE detector is presented in section 4 .

\section{GRAINE 2015 Experiment}

\subsection{Apparatus}

The aim of the GRAINE 2015 experiment was to demonstrate the flight performance of the middle-scale telescope. The payload consisted of the emulsion telescope, star cameras, and a pressure vessel gondola [21]. Figure 1 shows a cross-sectional view of the emulsion telescope. The components of the emulsion telescope were a converter, which stacked 100 emulsion films and detected pair creation events, a timestamper, which assigned incident time to each track using the multi-stage shifter technique [22], and a calorimeter, with a sandwich structure of emulsion films and metal plates, to determine the gamma-ray energy in the $\mathrm{GeV}$ region. The emulsion films with emulsion layers on both sides of a $180-\mu \mathrm{m}$-thick plastic film were used for the telescope. The thicknesses of the emulsion layers for the converter and timestamper were 70 and $50 \mu \mathrm{m}$, respectively. The star camera system monitored the attitudes of the emulsion telescope during the observation. Three far infrared cameras equipped with hoods to prevent stray light were placed on the gondola, to continuously capture and store the images of stars.

\subsection{Balloon flight}

The balloon flight of the GRAINE 2015 experiment was performed on May 12, 2015. The balloon carrying the payload was launched from the Alice Springs Balloon Launching Station, Australia, at 6:33 Australian Central Standard Time (ACST). It reached an altitude of $37.2 \mathrm{~km}$ at 08:50 ACST following ascent, and then the observation at the level flight started. The balloon floated at an altitude range of 36.0-37.4 km, heading eastward. The instruments were shut down at 20:00 ACST, and then the gondola was released at 20:22 ACST. The payload landed on the ground approximately $130 \mathrm{~km}$ north of Longreach at 20:55 ACST, as expected. The recovery team arrived at the landing site in the next morning, and a quick recovery was performed. All emulsion films were transported to Sydney and the photo-finishing process (development) was completed at the University of Sydney by June 1, 2015.

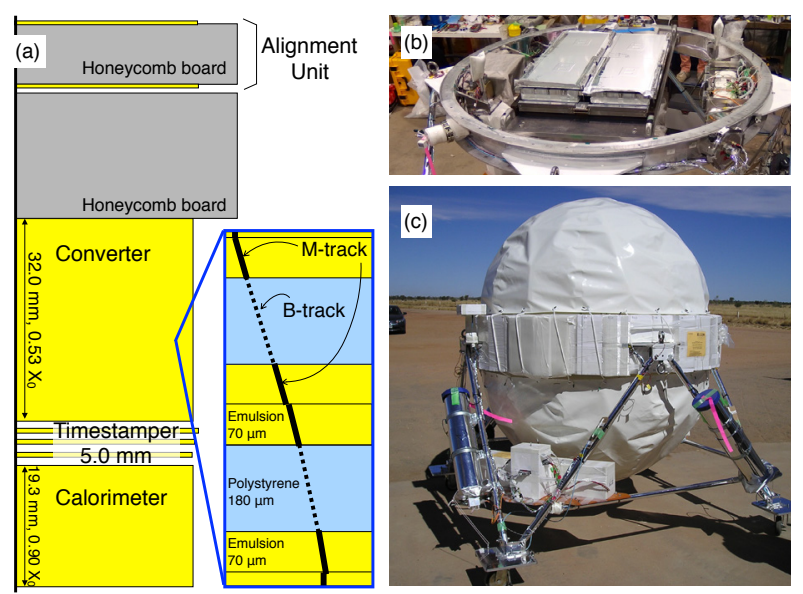

Figure 1. (a) Cross-sectional view of the emulsion films and the emulsion gamma-ray telescope. The telescope consists of an alignment unit, converter, time-stamper, and calorimeter. (b) Inside view of the pressure vessel. The emulsion gamma-ray telescope was placed at the center of a ring. The total aperture area of the four chambers was $3780 \mathrm{~cm}^{2}$. (c) Picture of the gondola. The spherical container (inside diameter of $1.6 \mathrm{~m}$ ) is a balloon-style pressure vessel. The three star cameras used as attitude monitors are deployed on the truss structure (one of them was on the other side) [20].

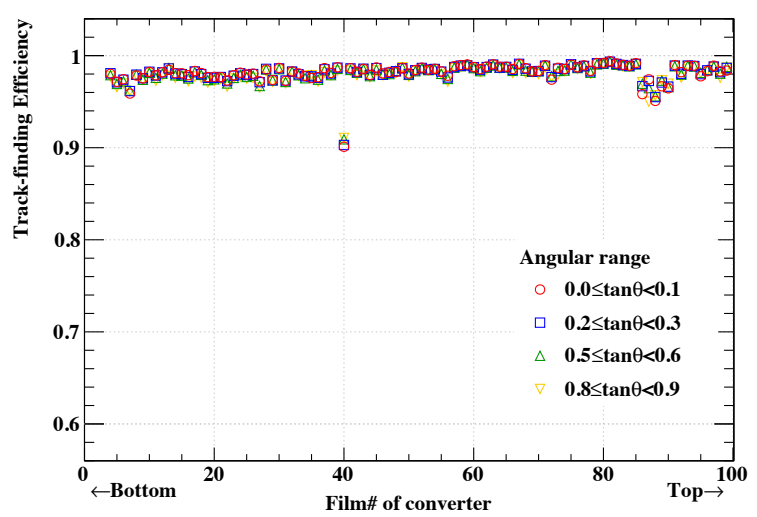

Figure 2. Track-finding efficiency as a function of the film number of the converter (unit \#3) [20].

\subsection{Data acquisition}

The tracks recorded in the flight emulsion films were scanned by the Hyper Track Selector (HTS) readout system, which was developed at Nagoya University [23]. The data acquisition of the converter and timestamper films was performed for approximately 3 months and HTS read a total film area of $41.3 \mathrm{~m}^{2}$. The quality and performance of the scanned data were analyzed by an off-line process. Figure 2 shows the track-finding efficiency determined for each film. A very good efficiency (greater than 95\%) was stably obtained for nearly all films. 

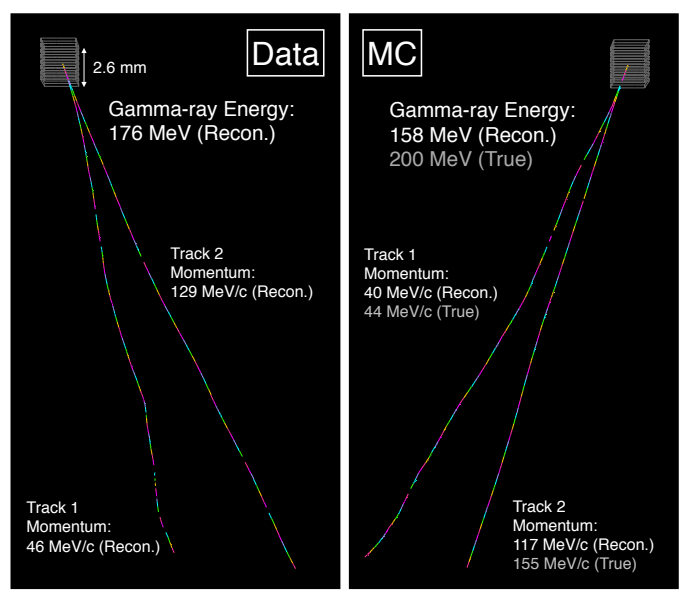

Figure 3. One of the gamma-ray events $\left(\gamma+(\gamma) \rightarrow \mathrm{e}^{+}+\mathrm{e}^{-}\right)$ detected in the GRAINE 2015 converter (left), and a simulated event (right) [20].

\subsection{Analysis}

\subsubsection{Selection of gamma-ray events}

The gamma-ray events were selected from the converter data obtained by HTS. The pair creation events start in the center of the converter, penetrate downstream, and are accompanied by a partner track. This selection process using the topological features is described in detail in Ref [20].

The three-dimensional event view shown on the left pane of Figure 3 is one of the selected events from GRAINE 2015 converter data. The tracks started at film \#95 (95th film from the bottom of the converter) and reached film \#1. The energy of the gamma ray was reconstructed from the momenta of the pair tracks, which were measured by the multiple Coulomb scattering method. In the case of this event, track \#1, which penetrates with a greatly varying angle, has a reconstructed momentum of $46 \mathrm{MeV} / \mathrm{c}$, and track \#2, which shows less variations of its angle, has $129 \mathrm{MeV} / \mathrm{c}$. The energy resolution was estimated to be $15-25 \%$ in the energy region of $50-300 \mathrm{MeV}$ using Monte Carlo (MC) simulation.

Automatic selection and energy reconstruction were preformed for $75 \%$ of the converter films employed in GRAINE 2015, and $2 \times 10^{6}$ gamma-ray events were acquired.

\subsubsection{Timestamp}

Timestamp analysis using a multi-stage shifter technique is described in Ref [24]. The tracks in film \#1 of the converter were connected to the films of the timestamper and incident time were assigned to them. Figure 4 shows the count rate of the charged tracks as a function of time obtained by the multi-stage shifter. The red line on the right axis indicates the altitudes of the balloon gondola. The region at the altitude of approximately $20 \mathrm{~km}$ corresponds to the Pfotzer maximum, where the maximum of count rate was observed.

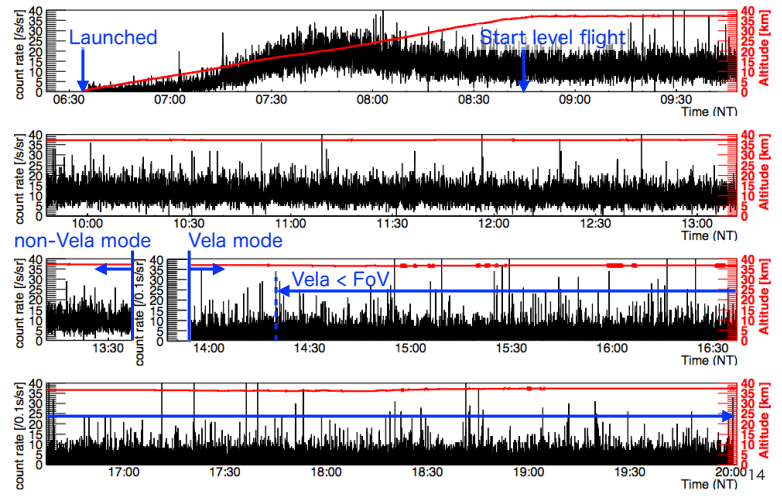

Figure 4. Count rate of charged tracks as a function of time obtained by multi-stage shifter. Red line on the right axis indicates the altitudes of the balloon gondola [24].
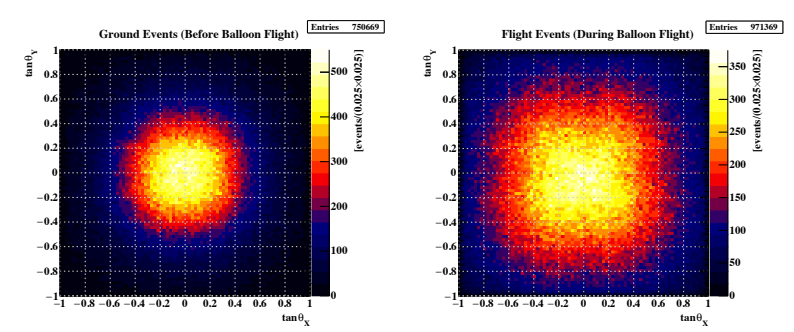

Figure 5. Angle distributions of gamma rays accumulated in the converter. The origin of the angles in the detector coordinates is nearly equal to the direction of the zenith. The left figure show the ground events, which were incident before the balloon flight; the right figure shows the flight events, which were incident during the balloon flight [20].

The operation of the multi-stage shifter was changed to the high-resolution mode, called Vela mode, at 13:45 ACST. During this period, the operation cycle of the stages became faster, to improve the time resolution. In the count rate distribution, many spiky structures can be observed. Such structures indicate that secondary tracks from hadronic interactions occurred in the converter. We evaluated the time resolution of the multi-stage shifter using these simultaneously generated tracks. Thus, the time resolution was evaluated in the range of 10-20 ms. This demonstration suggests that emulsion telescopes can realize phase-resolved observation of gamma-ray pulsars in future.

Figure 5 shows the angular distributions of gamma rays in detector coordinates. The origin of the angles is nearly parallel with the zenith direction. The gamma-ray events were divided into ground and flight events according to results of the timestamp. The ground events were recorded before the launch of the balloon and were expected to be generated via muon bremsstrahlung. Therefore, the distribution shows a strong dependence on the zenith angle. 


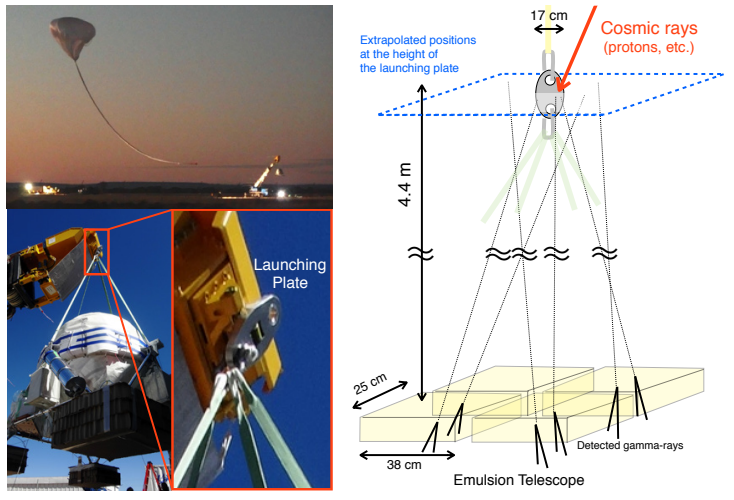

Figure 6. Photographs of the balloon, the crane truck, the gondola, and the launching plate (left). Schematic view of the positional relationship between the emulsion telescope and the launching plate (right). The square drawn with blue broken lines indicates a plane at the height of the launching plate [20].

\subsection{Demonstration of imaging using emulsion telescope}

We performed a demonstration of the gamma-ray imaging at balloon altitudes using the acquired gamma-ray data. Figure 6 shows the gondola suspended by a crane truck and an aluminum plate, called the launching plate. The plate connected the payload to a huge balloon. Following the launch, the plate was located $4 \mathrm{~m}$ above the emulsion telescope. When cosmic rays, mainly protons, interact with the plate, secondary particles including gamma rays are created. Thus, the launching plate became an external gamma-ray source for the calibration of the emulsion telescope during the flight.

The left pane of Figure 7 shows a gamma-ray imaging result of the launching plate using the emulsion telescope during the flight. A clear excess and the structure with the same size as the plate can be seen in the extrapolated position space, at the height of the launching plate. The histogram in the right pane of Figure 7 shows the $R^{2}$ distribution, where $R$ is the radius from the center of the excess.

We checked the expansion of the gamma-ray image using the prediction by the MC simulation. The four lines indicate the simulated distributions obtained by smearing the gamma-ray angles with angular resolutions $\theta_{68 \%} \times a$, where $\theta_{68 \%}$ is the designed angular resolution value of the emulsion telescope and $a$ is a constant value ( 0.0 for gray, 1.0 for red, 1.5 for blue, and 2.0 for green). The observed distribution shows good agreement with the prediction at $a=1.0$. So far, we have demonstrated the gamma-ray imaging performance using accelerator gamma-ray beams etc. in a small area in the emulsion chamber. This result is the first demonstration considering the entire enlarged aperture area of a balloon-borne emulsion telescope using an external gamma-ray source.

\section{Report on the GRAINE 2018 experiment}

In the GRAINE 2015 experiment, the instruments were upgraded in terms of both scale and performance. As a result, many improvements were achieved compared with the previous experiment (GRAINE 2011). Nevertheless, there were also some partial defects during observation, and the original aim of the experiment (the first detection of a celestial gamma-ray source) was not achieved. Thus, we devised an experiment that is an improvement of the GRAINE 2015 and have prepared a payload since 2017. The size of the detector was nearly the same, but we improved the detectors by considering the results from GRAINE 2015 to achieve a robust system and increase gamma-ray yields. The aim of this experiment is to demonstrate the overall performance of the emulsion telescope by detecting a celestial gamma-ray source (the Vela pulsar) in the 100-MeV energy region.

Collaborating GRAINE members arrived in the Alice Springs Balloon Launching Station, Australia on March 1, 2018. The assembly of the payload, operation test, and final compatibility check were completed by March 30. On April 26, the wind speed and direction predictions satisfied the required conditions, and the balloon launch was performed at 6:33 ACST. The balloon reached an altitude of $38 \mathrm{~km}$ in $2 \mathrm{~h}$ and started level flight heading eastward, similarly to GRAINE 2015. The emulsion telescope and the three star cameras were operating stably and the observation continued until 22:19 ACST. The Vela pulsar crossed a field of view of the telescope for approximately $7 \mathrm{~h}$ and the observation was successfully performed as planned. The balloon released the gondola equipped with a parachute at 23:17 ACST, which landed on the ground $250 \mathrm{~km}$ southwest of Longreach at 23:54 ACST. The recovery of the payload was completed using a helicopter on the next day, and the emulsion films were transported to Sydney immediately. The development of all films was processed at the University of Sydney. All processes were finished by May 13.

Currently, the data acquisition is performed and the data analysis is in progress.

\section{Hadronic interaction observation test at SPS/CERN}

In this section, we describe a beam test for the analysis of hadronic interactions, which is one of the groundbased tests carried out in parallel with the balloon experiment. As mentioned above, gamma rays emitted from hadronic interactions occurred in observation altitudes are very useful calibration sources. In particular, in the case of the events in the converter, the incident direction of secondary gamma rays can be determined because vertex points are precisely known. The evaluation of the angular resolution for gamma rays using such events were conducted in the analysis of GRAINE 2015 [25]. Nevertheless, secondary gamma rays become background for cosmic gamma-ray observations, and a study of the detection method for such hadronic interaction events recorded in balloon-borne emulsion detector is also important.

In 2016, we inserted the emulsion chamber, with the same structure as the GRAINE 2015 converter, into the beamline of the CERN SPS, and subjected it to $400 \mathrm{GeV} / \mathrm{c}$ 

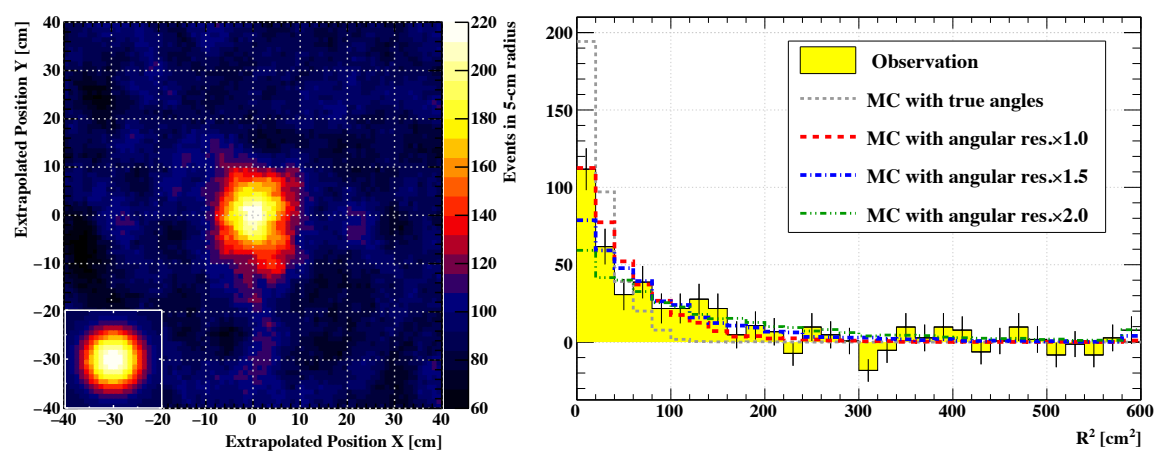

Figure 7. Extrapolated position distribution (left) as a result of counting events with running bins (5cm- radius circles). The energy range is set as $100-300 \mathrm{MeV}$. The inset shows the simulated point-source image in the energy region considered. Observed and MC-simulated distributions of $R^{2}$ after subtraction of the background contribution estimated by counting in off-source regions (right) [20].

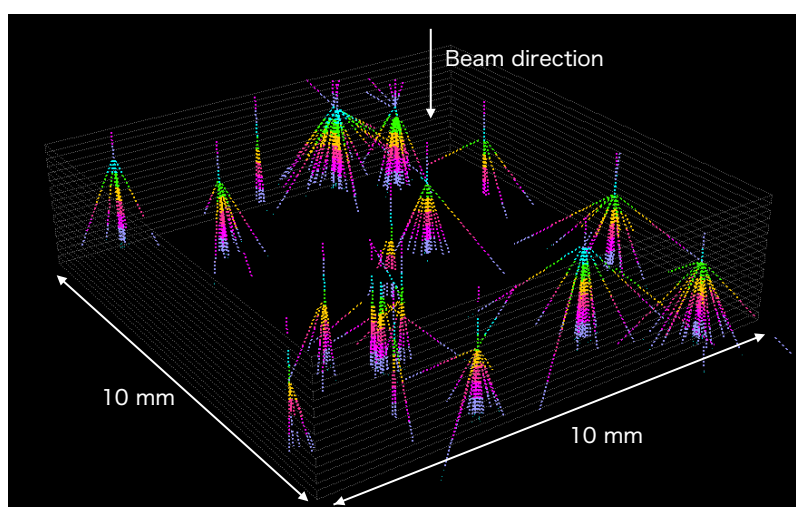

Figure 8. Three-dimensional view of $400-\mathrm{GeV} / \mathrm{c}$ protonemulsion interaction events. White square indicates a film area of $10 \mathrm{~mm} \times 10 \mathrm{~mm}$.

protons. Following the development process, data acquisition was conducted by HTS. Events with a vertex consisting of three or more tracks were selected using the reconstructed tracks. As a result, 72 candidates were selected in $20 \mathrm{~mm} \times 20 \mathrm{~mm} \times 0.7 \mathrm{~mm}$ volume of the analyzed volume. Figure 8 shows the three-dimensional view of the hadronic interaction events selected from an area of 10 $\mathrm{mm} \times 10 \mathrm{~mm}$. The number of beam tracks in the upstream film of this analysis region was $6.7 \times 10^{4}$ and the expected number of interactions was calculated at 87 events from the interaction length. Therefore, the selection efficiency by the current selection algorithm was determined to be $82 \pm 12$ (stat.)\% [26]. (The expected value from the MC simulation is $91 \%$ )

The left pane of Figure 9 shows the multiplicity distribution, and the right pane shows the distribution of the angle with respect to the beam direction using the selected secondary charged particles The blue histograms indicate the predictions calculated by the post-LHC interaction model (EPOS-LHC). As all charged particles within an angle of $58^{\circ}$ from the beam direction were read by HTS, the evaporated particles were also considered in the sim-
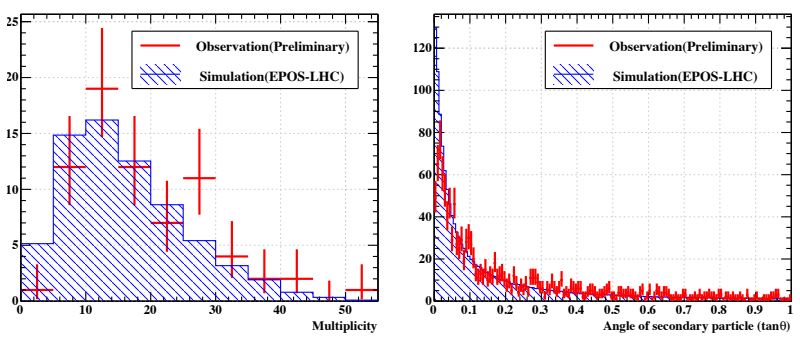

Figure 9. Distributions of multiplicity (left) and angle (right) with respect to the beam direction using secondary charged particles.

ulation data. Simulations were performed for each target (carbon, silver, bromide, and hydrogen) and the distributions were combined, considering the fraction of the number of expected interactions for each target. Both multiplicity and angular distributions were generally consistent with the predictions; however, a decrease in the number of tracks was observed in the angular region of $\tan \theta<0.04$. A possible reason for this is that HTS unites multiple tracks into a single track in the forward angular region; these details need to be further studied.

\section{Summary and Prospect}

GRAINE is a precise observation project of cosmic gamma-rays in the $0.1-100 \mathrm{GeV}$ energy region using balloon-borne emulsion telescopes. In the GRAINE 2015 balloon experiment, we developed a middle-scale telescope with an aperture area of $0.4 \mathrm{~m}^{2}$, and demonstrated gamma-ray imaging of a calibration source (the launching plate) in the $100-\mathrm{MeV}$ region and a time resolution of 10-20 ms. Nevertheless, the first detection of a celestial gamma-ray source was not achieved. Following various improvements of the detectors, we performed the GRAINE 2018 experiment. The balloon was launched on April 26, 2018 from Alice Springs, and then the observation, recovery, and development were completed as ex- 
pected. Currently, data acquisition is being performed. We aim to demonstrate the overall performance of the emulsion telescope by detecting the Vela pulsar in this balloon experiment. After this, we intend to start scientific observation with enlarged telescopes.

Simultaneously, detector studies on the ground were performed. Results in the proton beam test at SPS/CERN show that the nuclear emulsion with excellent spatial resolution enables the measurement of the topologies of hadronic interactions near vertex points. The plates used in the E653 experiment conducted at Fermilab in the 1980s, where the emulsion chamber was exposed to a $600-\mathrm{GeV} / \mathrm{c}$ $\pi^{-}$beam, have been stored. The verification for pionnucleus interaction models is possible by their review based on the latest emulsion readout system.

\section{Acknowledgements}

The scientific balloon (DAIKIKYU) flight opportunity was provided by ISAS/JAXA. This work was supported by JSPS, KAKENHI (grant numbers 17H06132, 18H01228, and 18K13562) and Nohmura Foundation for Membrane Structure's Technology.

\section{References}

[1] W. B. Atwood et al., Astrophys. J. 697, 1071 (2009).

[2] F. Acero et al., Astrophys. J. Suppl. Ser. 218, 23, (2015).

[3] M. Ackermann et al., Science 339, 807 (2013).

[4] T. Daylan et al., Phys. Dark Universe 12, 1 (2016).

[5] A. A. Abdo et al., Science 327, 1103 (2010).

[6] P. Gros et al., Astropart. Phys. 88, 30 (2017).
[7] A. Moiseev et al., PoS ICRC2017, 798 (2018).

[8] A. De Angelis et al., Exp. Astron. 44, 25 (2017).

[9] S. Takahashi et al., Adv. Space Res. 62, 2945 (2018).

[10] K. Ozaki et al., Nucl. Instrum. Meth. A 833, 165 (2016).

[11] S. Takahashi, PhD thesis, Nagoya University, Japan (2011) (in Japanese).

[12] H. Rokujo et al., PoS ICRC2015, 654 (2015).

[13] M. Morishita et al., PoS KMI2017, 073 (2017).

[14] Y. Nakamura st al., PoS KMI2017, 079 (2017).

[15] H. Rokujo et al., PoS KMI2013, 042 (2013).

[16] S. Takahashi et al., Prog. Theor. Exp. Phys. 2015, 043H01 (2015).

[17] H. Rokujo et al., Nucl. Instrum. Meth. A 701, 127 (2013).

[18] S. Takahashi et al., Prog. Theor. Exp. Phys. 2016, 073F01 (2016).

[19] K. Ozaki et al., J. Instrum. 10, P12018 (2015).

[20] H. Rokujo, et al., Prog. Theor. Exp. Phys. 2018, 063H01 (2018).

[21] H. Rokujo and N. Otsuka, PoS ICRC2015, 1021 (2017).

[22] S. Takahashi et al., Nucl. Instrum. Meth. A 620, 192 (2010).

[23] M. Yoshimoto et al., Prog. Theor. Exp. Phys. 2017, 103H01 (2017).

[24] F.Mizutani, et al. Transactions of JSASS, AEROSPACE TECHNOLOGY JAPAN 16.6, 464 (2018).

[25] H. Kawahara, PoS KMI2017, 059 (2017).

[26] M.Morishita, Master thesis, Nagoya University, Japan (2017) (in Japanese). 